$$
\begin{array}{r}
\text { April 26, } 1958 \\
I=\sqrt{m_{c} \omega_{p} \omega_{s} M_{p} / M_{c}^{t()_{B} k}}
\end{array}
$$

VOL. 181

\section{Importance of the Faraday to Elemental Constants and Electricity Standards}

RECENT values of the ratio $e / m$ as measured physically by deflexion in a magnetic field and as measured by electrochemical deposition or erosion permit combination in such a way that errors arising from uncertainties in the acceleration, $g$, due to gravity may be eliminated ${ }^{1}$. Work now in progress at the National Bureau of Standards on the gyromagnetic ratio (loc. cit., pp. 168-69) will permit an even more fortunate elimination.

In this new work the gyromagnetic ratio of the proton, $\gamma$, is being measured in a precision solenoid for which the coil constant can be calculated to an accuracy of a few parts in $10^{6}$, giving :

$$
\gamma=\omega_{s} / k I
$$

where $\omega_{g}$ is the precession frequency, $k$ the constant for the solenoid and $I$ the current in amperes. If the value of $\gamma$ so determined is used for measuring a magnetic field in which $e / m$ is to be determined by observing the cyclotron frequency of the proton, say, then we can express $e / m$ in coulombs per gm. for a singly-charged particle of unit atomic weight by :

$$
(e / m)_{p}=\omega_{p} \gamma M_{p} / \omega_{B}=\omega_{p} \omega_{8} M_{p} / \omega_{B} k I
$$

where $\omega_{p}$ is the cyclotron frequency of the particle, $\omega_{B}$ the precession frequency in the same field, and $M_{p}$ the mass of the particle in atomic units.

Determination of the Faraday by electrochemical deposition or erosion gives :

$$
(e / m)_{c}=M_{c} I t / m_{c}
$$

in which $M_{c}$ is the mass of the atom in atomic units of the substance involved in the process, $I$ the current in amperes, $t$ the time it flows, and $m_{c}$ the mass deposited or eroded. In this case $(e / m)_{c}$ is expressed in the same units as $(e / m)_{p}$.

Multiplying equations (2) and (3) yields

$$
e / m=\sqrt{(e / m)_{p}(e / m)_{c}}=\sqrt{\omega_{p \omega_{s} M_{p} M_{c} t / \omega_{B} k m_{c}}}
$$

Thus, the dependence of $e / m$ on the electricity standards is eliminated. All the quantities on the right-hand side of equation (4) can be measured with considerable accuracy. Since their major errors are uncorrelated, the error in $e / m$ is obtained by half of each individual error added in quadrature. The greatest errors are associated with $\omega p$, due to perturbing forces on the particles, with $m_{c}$, due to chemical impurities, and with $M_{c}$, if the substance used contains more than one isotope. There seem to be means available now whereby the uncertainties in these quantities may be reduced considerably with respect to what has been possible in the past, giving considerable improvement in accuracy of e/m.

The interesting aspect of this elimination is that with the more precisely known constants such as $R_{\infty}$ and $\alpha$, the Rydberg and fine-structure constants, $M_{e}$ the mass of the electron in atomic units, and $c$ the speed of light, the elemental constants $e, h, m$, etc., can be freed of uncertainties due to the electric standards and the acceleration of gravity. In adjustments to obtain the best values of these constants, the uncertainties in the electric standards and gravity are usually neglected, though not overlooked, in assessing the errors of the constants.

Conversely, dividing equation 3 by equation 2 yields : which gives the value of the current used in the experiments in absolute electric units, subject only to the errors of the quantities under the radical sign. This affords a determination of the accuracy of the electric standards used, quite independent of the errors involved in their experimental determination. Actually, the solenoid being used for the experiment for the determination of $\gamma$ is the same as that used for determining the ampere by the Pellat balance method (loc. cit., p. 153). However, the constant of the solenoid is so well known that its contribution to the error of that experiment is negligible.

\section{A. G. MCNisH}

R. D. Huntoon

National Bureau of Standards, Washington, D.C. March 5. 'Huntoon, R. D., and McNish, A. G., Il Nuovo Cimento, ser. 10,
Supp. 6, 146 (1957).

\section{Possible Existence of Anti-Matter in Bulk}

OF the many craters on the Earth known to have been produced by fallen meteors, a few have left no signs of the meteor which caused them, apart from the huge holes created in the Earth's crust. Large meteorite fragments have been found in or near most craters. A possible explanation of the lack of meteorite fragments in the other cases is that the meteors concerned were of contraterrene constituency (anti-matter). In this case no traces of the meteors would remain, due to the annihilation process. One of these events, from which no frag. ments appear to have survived, has occurred in recent years. In 1908 a meteor ${ }^{1}$, apparently of great size, was seen passing over western Russia ; a few minutes later it crashed in the Siberian wastes with an explosion, effects of which were felt for hundreds of miles around, and seismographs thousands of miles away registered its impact. Descriptions of its effects at the time indicated a release of power equivalent to that which might have been obtained from a thermonuclear explosion. No expedition to the site of its landing was undertaken until 1927, when the devastation found even at 40 miles from the site was almost inconceivable; but no fragments of the meteor were located despite the several huge though shallow eraters which it had caused. If this meteor consisted of anti-matter an obvious explanation is at hand for the devastation found, the lack of meteoric material, and the great size reported in eye-witness accounts of its passage.

It would therefore be of interest to re-examine the site with the view of searching for short-lived radioisotopes which would have been produced by the intense $\gamma$-radiation present. The presence of such short-lived isotopes (for example, aluminium-39, manganese-53, argon-39, titanium-44) would lend strong support to the hypothesis of anti-matter.

Department of Physics,

\section{Philip J. WyatT}

The Florida State University, Tallahassee.

Jan. 28.

1 cf. Watson, F. G., "Between the Planets" (Blakiston Co., Phila-
delphia, 1941). 